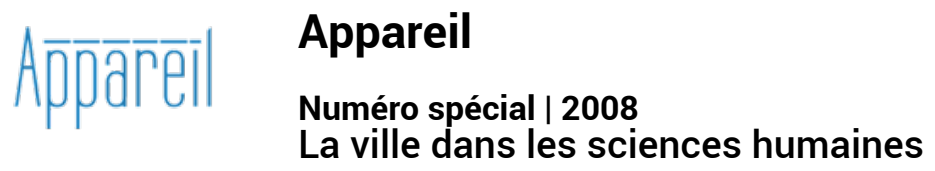

\title{
La Ville-Nature
}

\section{Chris Younès}

\section{(Q) OpenEdition}

Journals

Édition électronique

URL : http://journals.openedition.org/appareil/455

DOI : 10.4000/appareil.455

ISSN : 2101-0714

\section{Éditeur}

MSH Paris Nord

\section{Référence électronique}

Chris Younès, "La Ville-Nature », Appareil [En ligne], Numéro spécial | 2008, mis en ligne le 30 juin 2008, consulté le 30 juillet 2020. URL : http://journals.openedition.org/appareil/455 ; DOI : https:// doi.org/10.4000/appareil.455

Ce document a été généré automatiquement le 30 juillet 2020.

\section{(c) (i) () $\Theta$}

Appareil est mis à disposition selon les termes de la Licence Creative Commons Attribution - Pas d'Utilisation Commerciale - Pas de Modification 4.0 International. 


\section{La Ville-Nature}

\section{Chris Younès}

1 La ville, forme manifeste et archétypale d'un en-commun politique et spatial particulièrement emblématique avec la polis grecque, se trouve fortement ébranlée par l'essor de l'urbanisation qui coïncide avec des phénomènes sociaux à la fois de massification, de communautarisation et d'individualisation mais aussi avec de terribles dévastations écologiques, iniquités et fractures sociales. Les réorganisations par polarisation et extension qui multiplient et augmentent les déplacements reconfigurent les territoires. C'est du côté d'une proliférante nébuleuse d'espaces disparates souvent qualifiés de suburbains que se situe aussi la ville contemporaine dont Bernardo Secchi souligne le mixte :

Celle-ci ne résulte pas seulement de l'étape urbaine précédente, elle combine des éléments hérités et des métissages divers. Nous débattons actuellement sur l'opposition entre la «ville consolidée » et la "ville dispersée », entre celle qui protège la filiation historique, privilégie le lignage patrimonial, provoque une sensibilité au paysage et celle qui magnifie la dé-densification valorise les mobilités et, paradoxalement, provoque une sensibilité au paysage. Je me refuse à choisir entre ces deux types de ville, pour la simple raison que je crois qu'elles cohabitent, voisinent et parfois se combinent ${ }^{1}$.

2 Et la privatisation qui gagne du terrain rend cruciales tout autant les questions des limites que celles des partages.

Les évolutions ont de fait extrémisé une situation où chacun est sa «bulle ", contenue dans d'autres sphères, comme l'explique Peter Sloterdijk, depuis l'utérus jusqu'à l'internet en passant par la coupole et la ville, mettant l'accent sur la réalité d'un milieu hyper-relationnel paradoxal, une " écume » $\mathrm{de} \mathrm{vie}^{2}$. Dans La ville au loin, à propos de sa description de Los Angeles, Jean-Luc Nancy insiste sur l'importance d'explorer les expériences et les traversées de cet urbain contemporain sans le magnifier mais sans la nostalgie des villes du passé dont on reconstruit une vision idéale, comme si elles n'avaient pas été aussi le lieu de dominations, de séparations et d'enfermements :

La ville qu'on craint de perdre est la ville sans sa banlieue, celle que l'on craint est la ville avec banlieue et dans sa banlieue. Celle qu'on voudrait garder est la ville gouvernante et commerciale, la capitale bourgeoise qui se donne en tant que ville, 
la représentation de son pouvoir. Elle écarte sa banlieue et préserve son château, sa cathédrale ${ }^{3}$.

4 À la crise du vivre ensemble s'ajoutent les fortes préoccupations environnementales et écologiques. La thématique de la ville-nature fait écho aux transformations inouïes dans les façons d'appréhender les rapports non seulement entre ville et campagne mais également entre l'homme et son milieu de vie. L'expression de ville-nature associe des termes qu'il n'est pas habituel de rapprocher. Elle renvoie à des déplacements de plusieurs types, depuis la gestion de la présence physique de la nature en ville jusqu'aux changements paradigmatiques opérés en particulier avec Hans Jonas qui a insisté sur le principe responsabilité, Michel Serres sur le contrat naturel, Augustin Berque sur l'écoumène et Peter Sloterdijk sur la biosophie.

\section{Crise de la modernité}

5 L'art des établissements humains a toujours établi, au fil des millénaires, des rapports avec le milieu naturel, que ce soit en s'en tenant à distance par crainte ou respect, en tentant une imitation voire une symbiose ou bien en cherchant à le maîtriser jusqu'à une exploitation aveugle. La modernité occidentale des temps Modernes en est venue à opposer l'homme à la nature, suivant la représentation dualiste amorcée au XVII ${ }^{\mathrm{e}}$ siècle par Galilée, Bacon et Descartes, d'une nature extérieure à l'homme et qu'il pouvait manipuler à son gré. Cette conception est aujourd'hui remise en question, sous la double pression de la peur du monde ainsi produit ou dans un souci de responsabilité. À un imaginaire techniciste qui a orienté un certain modernisme, se superpose celui d'une symbiose et d'un ressourcement par le "naturel de la nature ", selon une expression heideggérienne :

Ce naturel de la nature ne se dégage pas directement de la nature même, on le reconnaît plus proprement dans ce qu'autrefois les anciens penseurs grecs nommaient la «physis » : l'éclosion/retrait de tout étant dans sa présence/absence. Le naturel de la nature, c'est ce lever et ce coucher du soleil, de la lune et des étoiles, qui s'adressaient directement aux hommes en leur révélant en quoi le monde est plein de son secret ${ }^{4}$.

6 L'irréductibilité de la nature qui hante les urbains est bien autre chose qu'une nostalgie de la vie villageoise ou un refus de la ville. Le désir de cette nature se développe en même temps que se généralise le monde urbain comme si les forces de la culture devaient se connecter à celles de la nature pour que le monde reste habitable. La nature ne peut être réduite à des métaphores, au verdissement, au végétal, à l'invocation de la campagne ou à des signes sérigraphiés. Avec le mot nature sont désignés l'eau, l'air, la terre, le feu, la faune, la flore... les rythmes des saisons, des jours et des nuits, du cœur et du souffle, de la veille et du sommeil ou de la naissance et de la mort... Mais le mot de nature renvoie d'abord à une genèse réitérée comme l'indique son étymologie latine natura (du participe futur de nascer) qui signifie «ce qui donne naissance, le fait de naître, ce qui présage de la chose », et qui correspond en partie au grec ancien physis dont la racine phù signifie "croître et pousser ». Aristote distinguait les êtres naturels des êtres fabriqués en ce qu'ils sont pourvus d'un principe d'automouvement et de repos, portant en eux-mêmes la possibilités de devenir autre, de se déplacer, de s'accroître ou de diminuer (Physique II, 1-192). La nature ne relève ni du domaine des choses extérieures à l'homme, elle est en nous (elle nous porte, considérait MerleauPonty dans son cours sur La nature au Collège de France), ni de l'univers qu'elle 
fabrique. Elle ne se réduit pas, même s'il en est une composante, au paysage, qui, en tant qu'espace perçu et artialisé est toujours déjà une construction mentale plus ou moins consciente d'une réalité qui s'offre à un sujet individuel et collectif. Elle ne se ramène pas non plus, même si elle en est une facette, à une approche environnementale visant le traitement scientifique de données factuelles. La polysémie de la nature, au nouage du réel, de l'imaginaire et du symbolique est indissociable de ses expressions culturelles en leur diversité. Elle semble se présenter à l'heure actuelle comme un antidote ou un potentiel de régénération dans un monde fortement marqué voire déséquilibré par l'artifice. Chacun peut constater avec Ricœur que "l'homme de la technique ajoute une fragilité supplémentaire qui est son œuvre». Il apparaît désormais que les capacités de prévision qui caractérisent le savoir scientifique se trouvent débordées par la croissance des incertitudes quant aux effets à court ou à long terme des interventions technoscientifiques susceptibles de se révéler catastrophiques pour les milieux habités.

\section{D’autres configurations des milieux habités}

7 L'inquiétude liée aux dévastations des écosystèmes et à la prise de conscience de la finitude de la planète Terre, de sa vulnérabilité et de celle des hommes ${ }^{5}$ amène à s'interroger sur les stratégies de transformation des milieux et sur les rapports soutenables à établir entre nature, technè et société. Un revirement est en jeu. Si être moderne, c'était privilégier la tabula rasa et s'affranchir du contexte, le défi consiste désormais à imaginer d'autres possibles par la mise en œuvre de synergies d'un autre type entre les dynamiques de la culture et celles de la nature. L'aspiration est forte non seulement à bénéficier dans son habitat urbain d'une mise en contact avec la nature mais également à trouver un juste positionnement entre nature et artefact pour se prémunir des catastrophes naturelles ou provenant de l'action humaine, mais aussi pour en capter les forces vivifiantes. Ainsi la ville d'Oslo est indélébilement connectée au fjord, à la forêt et aux collines nordiques, Bogota à la Cordillère des Andes, ainsi qu'à leur histoire spécifique.

8 Le développement durable (rapport Brundtland, 1987) qui préconise un développement viable, vivable, équitable, constitue un nouveau contexte international. Cette orientation qui s'est présentée à la fin $\mathrm{du} \mathrm{xx}^{\mathrm{e}}$ siècle comme un cadre contractuel planétaire, a pris naissance dans la conjonction des technosciences, du primat de l'économie financière, des préoccupations écologiques et de l'évolution des modes de vie. Au-delà des ambiguïtés et des polémiques qui sont soulevées à son propos, son impact dans l'élaboration de projets de devenir urbain est en cours. Il conduit, pour transformer les milieux à habiter, à privilégier d'autres types de " prises ", suivant une expression de Merleau-Ponty reprise par Augustin Berque. Trois sont particulièrement significatives :

- l'abandon de modèle et la revalorisation de l'art de s'adapter aux situations locales (résistances et résonances) en prenant en compte les enjeux et effets de la globalisation;

- l'affirmation dans un monde technique et marchand de la question politique et du positionnement éthique par rapport à toute décision de transformation ;

- l'invention de dispositifs capables d'articuler différentes échelles problématiques et spatiotemporelles dans une architecture des milieux, correspondant non à des figures territoriales 
ou à des formes urbaines a priori mais à des interrelations et des équilibres dynamiques mettant en jeu des modalités de gouvernance et des reconfigurations.

Les limites et passages entre ville et nature constituent avec le souci du vivre ensemble des chantiers déterminants. Natures, paysages et vides se trouvent désormais au cœur de stratégies urbaines régénératrices, que ce soit dans les territoires périurbains distendus ou dans les villes compactes. En tirer parti suppose un changement radical de mode de pensée, de gestion et d'invention du territoire comme nous l'avons analysé à l'occasion de différentes recherches menées au sein du laboratoire Gerphau ${ }^{6}$. Plusieurs figures entre nature et ville se détachent, que Frédéric Bonnet formule ainsi :

- la nature en ville comme facteur qualitatif de la vie urbaine : pouvoir profiter d'un jardin, de frondaisons forestières, d'éléments naturels tels que soleil, ombre, vent, paysage...

- le potentiel géographique. Toute ville est corrélée à un lieu fondateur à la géographie singulière. Ainsi Paris est construite sur une topographie, des collines, des lignes d'eau qui lui donnent par devers les siècles sa forme d'ensemble. Lorsque cette géographie a l'ampleur d'un bord de mer ou d'une rive de fleuve, le potentiel pour le développement urbain est précieux : pouvoir densifier tout en libérant les horizons. Le bord de l'eau prend aujourd'hui une forte valeur positive. Il devient limite épaisse habitée.

- les lisières entre ville et campagne. Comment stabiliser la limite entre ville et espace agricole et inverser le mouvement de l'étalement urbain où les champs ne sont plus que des réserves foncières implicites pour la croissance urbaine? Une telle orientation suppose de traiter des champs et forêts comme parts actives de l'économie et du paysage de la métropole, à savoir en termes d'activités, usages, promenades, narrations...

- le parc, jardin habité ; figure qui vise à lutter contre l'étalement pavillonnaire en alliant densité, équilibre et mise en contact avec paysage et nature.

Dans une analyse que nous avons menée des projets d'Europan ${ }^{7}$ primés lors des sept sessions qui se sont déroulées de 1989 à 2004, l'émergence de la thématique de la nature et de nouvelles postures architecturales visant à la capter, révéler, ménager, équilibrer, davantage qu'à la réduire à sa merci, est significative. Différentes figures ont pu être identifiées, qui caractérisent des types d'articulation entre nature et architecture et mettent en évidence le changement de paradigme en cours: déplacement d'une utilisation de la nature comme décor d'abord vers une nature de plus en plus considérée comme vecteur qualitatif d'un nouveau type d'espaces en tant que puissance dynamique avec laquelle l'architecture peut composer et se renouveler. Les propositions des équipes primées se trouvent en écho avec l'évolution sociétale qui s'exprime à travers de nombreux textes, expositions, débats à propos du désir de nature et des préoccupations quant aux milieux de vie des humains. En dix-huit ans, on est passé d'un programme strictement architectural, centré sur les innovations de cellules et de leurs assemblages à une réflexion sur les alentours et la ville habitée. Dans le même temps, les candidats ont été confrontés à des sites périurbains dont le périmètre est de plus en plus vaste jusqu'à des sites de grands territoires, avec une forte présence d'éléments naturels géographiques. Le concept «d'urbanoarchitecture ", devient un élément structurant : le sujet est aussi bien l'architecture que le paysage, l'infrastructure et l'environnement. Bien que des dominantes puissent être notées, ces évolutions des figures manifestent également des inerties, des récurrences, des sédimentations, des combinaisons. Elles ne sont pas non plus associées à un seul projet ou à un seul texte mais sont sous-jacentes. Elles expriment des postures 
ou des schèmes opératoires antagonistes, dont nous avons repéré cinq principales modalités caractéristiques :

- l'architecture donne le cadre de la nature considérée comme un décor assujetti aux règles disciplinaires de l'objet architectural ;

- l'architecture et la nature sont posées comme antagonistes ;

- les références sont hybridées comme réinterprétation des modèles d'urbanisme paysagers (cités-jardins, « parkways »,...);

- l'opposition plus ou moins latente entre artefact et nature s'estompe au profit d'alliances sous toutes leurs formes (textures et tissages, fondations géographiques, forces des éléments naturels tectoniques, atmosphériques et biologiques), que ce soit par des thématiques à nouveau présentes telles que l'agriculture comme matrice de l'urbain, le génie écologique ou les projets d'une nature domestique ou sauvage comme partie intégrante de l'urbanité ;

- le potentiel de la nature sauvage comme ressource et milieu du vivant, très rarement utilisé en tant que tel, est souvent convoqué pour illustrer les processus de croissance, les dynamiques de transformation et leurs combinatoires: ces références presque vitalistes rappellent simplement que la nature fascine par les puissances qu'elle convoque mais aussi par les diversités qui, dans un ordre pourtant bien déterminé et selon des structures identifiables, surgissent toujours de ses combinaisons. Le désir conjoint de maîtrise et d'aléas, d'ordre et de variété infinie se cristallise ainsi dans des métaphores naturalistes.

11 Les pratiques et recherches en architecture des territoires et des milieux urbains qui prennent au sérieux le vivant capable de reconfigurer son milieu ${ }^{8}$, et réciproquement, aboutissent à la création de nouvelles configurations. P.Sloterdijk explique les enjeux anthropologiques, politiques, topologiques en termes à la fois d'un système de représentation qui prend fin et d'amorce d'un autre, dans lequel la question des lieux et des situations de vie est déterminante :

La philosophie en tant que forme de pensée et de vie de l'ancienne Europe est indéniablement épuisée; la biosophie vient tout juste d'entamer son travail ; la théorie générale des systèmes immunitaires et ses systèmes communs en est à ses débuts ; une théorie des lieux, des situations, des immersions se met timidement en marche'.

Ce philosophe envisage l'espace humain et son évolution en explorant les appareillages de l'anthropisation de la terre à partir des métaphores des sphères et leur passage à celles des écumes. En philosophie comme en architecture, un défi est ouvert : penser un repositionnement éthico-politique du rapport entre nature et culture, technique et société, ville et milieux.

\section{BIBLIOGRAPHIE}

Bonnet Frédéric, Younès Chris, La nature et les projets : enjeux et figures, Europan France, 2006.

Canguilhem Georges, « Le vivant et son milieu », in La connaissance de la vie [Hachette, 1952], Paris, Vrin, 1998.

Goetz Benoît, La Dislocation, Paris, Éditions de la Passion, 2001. 
Heidegger Martin, « Hebel, l'ami de la maison » [1958], Questions III, Julien Hervier (trad.), Paris, Gallimard, 1966.

Nancy Jean-Luc, La ville au loin, Paris, Mille et une nuits, 1999.

Sloterdijk Peter, Sphères I, II et III, Écumes, Sphères III [2003], Paris, Maren Sell éditeurs, 2005.

\section{NOTES}

1. Bernardo Secchi, revue Urbanisme, $\mathrm{n}^{\circ} 306$, p. 12.

2. Peter Sloterdijk, Sphères I, II et III, Écumes, Sphères III [2003], Paris, Maren Sell éditeurs, 2005.

3. Jean-Luc Nancy, La ville au loin, Paris, Mille et une nuits, 1999.

4. Martin Heidegger, « Hebel, l'ami de la maison » [1958], Questions III, Julien Hervier (trad.), Paris, Gallimard, 1966, p. 58.

5. Voir la préface de Jean-Luc Nancy, dans l'ouvrage de Benoît Goetz, La Dislocation, Paris, Éditions de la Passion, 2001.

6. Cf. notamment Échelles et temporalités - PUCA (2003-2006), Architecture de la grande échelle DAPA-BRAUP (2007-2008), Analyse des projets d'Europan de 1989 à 2004.

7. Frédéric Bonnet, Chris Younès, La nature et les projets : enjeux et figures, Europan France, 2006. Europan est un concours à partir de situations urbaines proposées par des villes européennes, s'adressant aux jeunes architectes et concepteurs de toute l'Europe.

8. Georges Canguilhem, «Le vivant et son milieu », in La connaissance de la vie [Hachette, 1952], Paris, Vrin, 1998.

9. Peter Sloterdijk, Écumes, Sphères III, p. 19.

\section{AUTEUR}

\section{CHRIS YOUNÈS}

Philosophe, elle dirige le laboratoire CNRS du Gerphau. 